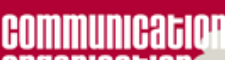

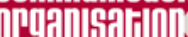

Communication et organisation

Revue scientifique francophone en Communication organisationnelle

$60 \mid 2021$

Influence et organisations : cultures, pratiques et mises en perspective

\title{
Pour une approche communicationnelle de l'influence
}

For a communicative approach to influence

Camille Alloing, Benoît Cordelier et Stéphanie Yates

\section{OpenEdition}

Journals

Édition électronique

URL : https://journals.openedition.org/communicationorganisation/10385

DOI : 10.4000/communicationorganisation. 10385

ISSN : $1775-3546$

Éditeur

Presses universitaires de Bordeaux

Édition imprimée

Date de publication : 1 décembre 2021

Pagination : 11-20

ISSN : 1168-5549

\section{Référence électronique}

Camille Alloing, Benoît Cordelier et Stéphanie Yates, « Pour une approche communicationnelle de l'influence », Communication et organisation [En ligne], 60 | 2021, mis en ligne le 05 janvier 2022, consulté le 15 juin 2022. URL : http://journals.openedition.org/communicationorganisation/10385 ; DOI : https://doi.org/10.4000/communicationorganisation.10385 


\title{
Éditorial
}

\section{Pour une approche communicationnelle de l'influence}

\section{For a communicative approach to influence}

\author{
Camille Alloing, Benoît Cordelier, Stéphanie Yates
}

L'influence comme objet de recherche en sciences sociales, et en communication en particulier (Mucchielli 2009), mobilise de nombreuses définitions et approches, mettant en leur centre aussi bien les médias (Maigret 2015) que les interactions sociales (Katz et Lazarsfeld 1955). En première lecture, l'influence est un ensemble de " processus ordinaires par lesquels un individu ou un groupe parvient à faire accepter des manières de faire, de ressentir et de penser qui font normes et qui agissent sur les attitudes et les comportements d'autres individus et groupes " (Bernard 2015 : 47). Par un prisme plus instrumental, nous pourrions dire " qu'exercer une influence consiste à obtenir d'autrui qu'il fasse librement quelque chose qu'il n'aurait pas fait spontanément sans votre intervention " (Massé et al. 2006). L'influence peut, à l'inverse, se traduire par le statu quo, lorsque l'objectif est, par exemple, d'éviter toute mobilisation (Gendron 2014). L'influence est ainsi généralement abordée par un angle psychosociologique comme le résultat d'un acte de communication sur les comportements ou représentations sociales des publics (Jodelet 2003); comme le résultat d'un processus de mise en circulation des idées et des opinions (Heath 2006) ou encore comme un ensemble de pratiques et stratégies visant la persuasion (Breton, 2008 ; Fourquet-Courbet et al. 2013).

Que ce soit dans les recherches académiques ou dans les discours destinés aux praticiens, les travaux en marketing et en psychologie dominent les discussions sur l'influence comme objet que l'on pourrait théoriser, analyser, quantifier, manipuler, 
voire mettre en marché (François et Zerbib 2015 ; Dupont 2011 ; Cialdini 2009). De fait, les théories de l'influence peuvent-elles se départir de la psychologie et du marketing ? Et peut-on penser cette notion en dehors de toute visée instrumentale et des nombreux imaginaires de manipulation ou de contrôle qui l'accompagnent ? Ce numéro de Communication \& Organisation propose ainsi d'enrichir notre champ conceptuel en discutant d'influence par le prisme d'autres notions commela diplomatie, le lobbying, les pratiques numériques, la microcélébrité, et même la magie, afin de renouveler notre compréhension du concept. En trame de fond, ce dossier s'intéresse d'abord aux éléments de contexte, et particulièrement aux dimensions culturelles en tant que soubassement - ou infrastructure - de l'influence (Seltzel et al. 2013). Ainsi des éléments culturels, qu'ils soient liés aux pratiques, aux modes organisationnels ou plus largement aux valeurs sociétales, permettent à l'influence de s'exercer dans un contexte donné, et de générer des effets ou affects tangibles, de l'adhésion (ou de l'acceptation), de l'engagement ou encore de la mobilisation.

La place centrale du "numérique " (ses industries, dispositifs, usages, économies) dans les stratégies et pratiques communicationnelles amène là aussi à réinterroger constamment la notion d'influence, voire à essayer de la réactualiser pour mieux la cerner (Coutant 2013), ou encore pour en faire une prestation marchande au sein d'un marché de la réputation numérique (Alloing 2015). En s'intéressant aux pratiques de l'influence numérique, on observe que les dispositifs sociotechniques du web peuvent être complémentaires à d'autres médias (de masse ou non) en tant que vecteurs de l'influence. Mais ils génèrent aussi de nouvelles difficultés pour rejoindre les différents publics d'une organisation, parfois cantonnés dans des " chambres d'écho "(Colleoni et al. 2014) ou de " réfraction " (Rieder 2012). Ces pratiques professionnelles visant à produire ou instrumentaliser de l'influence, en particulier pour les relations publiques et la communication organisationnelle, sont ainsi en constante réorganisation (Desmoulins et al. 2018) et conduisent à une extension des régimes d'influence et de responsabilisation (Cordelier et Breduillieard 2011). Les indicateurs issus de la quantification de cette influence prolifèrent sur les plate-formes qui définissent leurs propres standards et concepts, mettant les praticiens dans une situation de dépendance aux standards de ces dispositifs (Alloing et Pierre 2019).

Les leaders d'opinion, que Katz et Lazarsfeld avaient définis il y a plus de 70 ans maintenant et que les travaux en marketing ont parfois eu tendance à réduire à leur seule expertise d'un marché (Feick et Price 1987), deviennent des "influenceurs". Plus que des producteurs d'opinions incontournables (Poell et al., 2016 ; Charest et al. 2017), ils sont à la fois des (micro)célébrités qui savent se médiatiser (Gräve 2017), des spécialistes des algorithmes des plate-formes avec lesquels ils jouent au quotidien (Cotte 2019) et des fins connaisseurs de leurs publics qui s'identifient à eux. Lorsqu'ils prennent la parole au nom d'une marque, ils favorisent la confiance dans les discours qu'ils mettent en circulation (Singh et al. 2020). Dans un contexte 
info-communicationnel où une même plate-forme permet autant la publicité que la désinformation, bousculant alors les codes de production légitime de la connaissance (Lalancette, Yates et Rouillard, 2020), les usagers des dispositifs numériques ayant un fort capital d'influence sont un moyen de légitimer certains messages ou certaines mobilisations. Qui plus est, cette influence sur les groupes en ligne amène à s'interroger sur la qualité et l'instrumentalisation du lien social (Cordelier et Turcin, 2005) qui en découlent. Dans tous les cas, les modèles autoritatifs prénumériques sont transformés (DiStaso et Bortree, 2014 ; Alloing, 2016). En somme, les pratiques d'influence déployées dans les espaces numériques produisent des comportements ou discours observables aux conséquences sociétales souvent fortement médiatisées, et dont il est nécessaire de mettre en perspective les réels effets qui en découlent et les processus qui les autorisent.

Pour autant, même s'il paraît plus évident d'identifier les changements dans les conditions et formes d'influence sur et par le numérique, les pratiques organisationnelles d'influence ne se limitent pas à ce médium. Les démarches d'influence classiques conservent ainsi pleinement leur pertinence, qu'il s'agisse de gestion de la réputation (Turbide 2017 ; Huffaker 2010), de lobbyisme (Koutroubas et Lits 2011 ; Juanals et Minel 2013), d'astroturfing (Lock et al. 2016), de mécénat ou de commandite (Cordelier et Desaulniers 2020), de publicité (Kapferer 1978) ou encore de relations de presse (Motulksy 2018) ou plus largement, de stratégies de médiatisation (Yates 2018 ; 2019). Or, pour être effectives, ces pratiques doivent être en phase avec les éléments de contexte mentionnés précédemment, tout en tenant compte des dynamiques qui ont cours dans les espaces numériques, mais qui ont une incidence directe sur leur déploiement (Hampton et al. 2017), et ce malgré la résistance de certains praticiens à se les approprier (Kondratov 2018). En somme, l'économie de l'influence, comme le travail communicationnel qu'elle suppose, est large et nécessite de mettre en perspective cette notion en fonction des contextes et dimensions culturelles où elle se déploie, autant que d'interroger les pratiques qui y sont associées pour devenir un levier des stratégies des organisations. Les nombreuses propositions envoyées par des chercheuses et chercheurs francophones pour participer à ce dossier de la revue Communication \& Organisation témoignent de l'intérêt d'étudier ce sujet plus en profondeur dans les sciences de l'information et de la communication.

Ce dossier est structuré autour de trois grands thèmes qui fédèrent les membres du Laboratoire sur l'influence et la communication (Labfluens) de l'université du Québec à Montréal ${ }^{1}$, à savoir l'influence comme un concept à mieux circonscrire (1) et comme un ensemble de pratiques communicationnelles (2), qui s'insèrent dans un marché dont les métiers évoluent en conséquence (3). Dans leur article «Agir par

1- Des collègues qui ont par ailleurs participé à la réflexion et l'élaboration de l'appel à articles : Vincent Fournier, Bernard Motulsky, et Olivier Turbide. 
l'invisible ", Hugo Souza de Cursi, Valérie Durieu et Piergiorgio Bruno développent un regard historique, aux XIX ${ }^{\mathrm{e}}$ et $\mathrm{Xx}^{\mathrm{e}}$ siècles, afin de mieux cerner cette notion. Souza, Durieu et Bruno émettent l'hypothèse que l'influence relève autant d'une forme de pensée " magique " que scientifique, la première venant combler l'absence de preuves nécessaires à la seconde pour asseoir la légitimité de ce concept. Cette tension produit alors un «bégaiement », entre une influence dont les effets relèvent autant de l'indicible que des tentatives de lui donner corps, de la rendre tangible, notamment par le recours systématique aux travaux en psychologie. Ce bégaiement est discuté via l'analyse de deux corpus constitués d'écrits interrogeant la magie et l'influence au cinéma (ouvrages de réalisateurs, de critiques, d'analystes, etc.) et dans les métiers de la publicité (autobiographies, manuel, etc.). Ce travail de généalogie de la pensée magique dans la construction des représentations de l'influence se conclut par la mise en relief de sa présence dans les discours contemporains : faire de l'influence consisterait à manipuler des liens invisibles, comme par magie. Les analyses proposées dans cet article sont une invitation à ne plus faire l'économie de la généalogie de notions flottantes comme l'influence afin de mieux appréhender leurs usages actuels, et à effectuer un travail interdisciplinaire en sciences sociales et humaines pour arriver à cela.

Cette volonté de ne pas se limiter à une définition de l'influence relevant parfois d'un allant de soi, et aux imaginaires nombreux, se retrouve dans les autres articles présents dans ce numéro. En particulier, et toujours au niveau de la conceptualisation de cette notion, Lucile Desmoulins propose, là encore à partir de l'étude de textes, d'interroger la définition du "lobbying " par les métiers de l'influence. L'autrice remet en question la ligne de démarcation traditionnellement tracée entre lobbying et plaidoyer, ou entre la défense d'intérêts privés et de causes qui relèverait de l'intérêt général, en faisant valoir qu'on assiste bien, dans les deux cas, au déploiement de stratégies d'influence aux visées politiques. L'ère numérique qui caractérise le contexte contemporain serait d'ailleurs propice au développement créatif de nouvelles pratiques qui contribuent encore à brouiller la distinction entre ces démarches d'influence. D'ailleurs, l'autrice fait valoir que le brouillage de cette ligne de démarcation est parfois le fruit du travail délibéré de porteurs d'intérêts privés, qui chercheraient ainsi à valoriser l'image même du lobbying en l'associant à des démarches souvent de nature informative, dont les motifs seraient respectueux du droit, de valeurs morales et de considérations éthiques. Elle ajoute que le fait que les divers porteurs d'intérêt se réclament parfois des démarches de grassroot lobbying illustre bien la complexité de cette dynamique, alors que l'instrumentalisation des mouvements citoyens, voire leur création de toutes pièces, reste un élément difficile à cerner, comme l'illustrent les recherchent sur l'astroturfing. Les slapp (strategic lawsuit against public participation), le soft power et le plaidoyer d'entreprise sont d'autres types d'intervention qui rapprochent, encore une fois, le lobbying tel que traditionnellement conçu des démarches de plaidoyer. Au final, ces nombreuses convergences ont des répercussions 
concrètes sur les pratiques de l'influence. Cela nous amène aux deux autres thèmes de ce numéro : les pratiques et les métiers.

Les pratiques présentées dans les articles de ce numéro s’inscrivent dans différents contextes, tant nationaux qu'internationaux, numériques que médiatiques, et façonnent les métiers qui les emploient, comme elles sont façonnées par les contextes où elles se déploient. Josianne Millette et Alexandre Coutant développent ainsi dans leur article une approche socio-technique des métiers de l'influence. Leur proposition permet d'aller au-delà des promesses de l'influence pour observer les modalités de production et les contraintes propres aux métiers des relations publiques qui font de l'influence un produit à vendre aux annonceurs et une finalité de leurs actions. Associant deux observations de pratiques professionnelles numériques, en France et au Québec, Millette et Coutant soulignent l'importance de développer un programme de recherche s'intéressant aux effets des dispositifs socio-techniques sur les représentations de l'influence, à la façon dont ces mêmes dispositifs orientent les usages des praticiens, et enfin comment ces représentations et prescriptions encadrent les pratiques qui fabriquent l'influence proposée comme une prestation. Leur travail montre que loin d'être un objet stabilisé, l'influence telle qu'elle se donne à voir dans les environnements numériques est le résultat de négociations constantes entre différents acteurs, et de multiples adaptations aux situations sur lesquelles ces acteurs cherchent à avoir une emprise.

Ces négociations et adaptations, comme ce que les pratiques d'influence changent pour certains métiers, notamment avec l'apport du numérique, qui crée de nouvelles occasions, sont abordées par l'entremise de deux autres articles. D'abord, l'article de Simona De Iulio, Patrice de la Broise, Laurence Depezay et Susan Kovacs permet une mise en perspective des pratiques de microcélébrités dans le domaine de l'alimentation. Les auteurs nous montrent comment une certaine spectacularisation ou mise en scène de ce qui aurait dû être une banalité quotidienne se transforme en pratiques d'influence qui acquièrent une valeur marketing susceptible d'intéresser des annonceurs. La question de la proximité se récupère d'un certain éloignement sémantique vers lequel les études sur les leaders d'opinion des décennies précédentes nous avaient amenées.

Enfin, dans son article intitulé «La communication façon loup combattant : une stratégie d'influence de l'ambassade de Chine en France à l'ère de la pandémie de Covid-19 ", Zhao Alexandre Huang s'intéresse au travail des diplomates chinois en France en vue de restaurer l'image de la Chine. Alors que cette dernière est critiquée pour sa gestion lacunaire des débuts de la pandémie, marquée par la censure et le manque de transparence, l'auteur montre, via une fine analyse des nombreux tweets émis par l'ambassade de Chine en France dès les débuts de la pandémie, comment la diplomatie chinoise déploie une stratégie d'influence qui vise à réparer cette image négative. Par la mise en avant de discours et d'un récit qui combine les aspects 
défensifs et offensifs, inspirés de la " guerre prolongée " de Mao, les diplomates chinois insistent sur l'action responsable des dirigeants chinois, tout en déployant une réponse agressive aux attaques des pays occidentaux à l'endroit de la Chine - d'où l'image du loup combattant, totalement assumée par les dirigeants chinois. Au final, cette stratégie permet aux diplomates de présenter la Chine en tant que grande puissance mondiale qui a su déployer un modèle de prévention efficace lié à la pandémie de Covid-19.

En définitive, ce numéro n'amène pas de définition communicationnelle de l'influence. Et c'est tant mieux! Il se recentre sur des faits observables et documentés, qui relèvent de manières de faire ou de dire, plus que constructions conceptuelles. L'influence est opératoire, elle s'inscrit dans une vision praxéologique de la communication des organisations car elle répond à la question : communiquer pour quoi faire ? Dès lors, elle oscille, lorsqu'elle est mise en perspective par des approches communicationnelles, entre une notion-écran telle qu'employée par les praticiens des organisations, c'est-à-dire comme un objectif à atteindre et l'évaluation de l'atteinte de cet objectif, et comme un objet de recherche qui permet de s'intéresser à des pratiques, à des productions (écrites, visuelles, etc.), à des contextes et situations de communication, mais aussi à certaines idéologies ou normes. Loin d'être réductible à sa seule conception cognitive, l'influence est ainsi un concept qui ouvre la voie à de nombreuses recherches en communication des organisations et au-delà, pour l'étude des relations et interactions entre divers acteurs humains, organisationnels, médiatiques et techniques.

\section{Bibliographie}

ALLOING Camille, 2015, "Votre entreprise plus nette sur le Net. Comment les professionnels fabriquent-ils les publics et les imaginaires de l'e-réputation ?", Communication. Information médias théories pratiques, 33, 2.

ALLOING C., 2016, La E-réputation. Médiation, calcul, émotion : médiation, calcul, émotion, Paris, CNRS.

ALLOING Camille \& PIERRE Julien, 2019, «Une approche praxéologique des métriques numériques : mesurer le community management pour quoi faire? "Communication \& Professionnalisation, 9, 85-108.

BERNARD Françoise, 2015, " La communication des organisations entre questions d'influence et questions d'autonomie. L'actualité des notions d'engagement, d'émergence et d'institution ", Communication \& Organisation, 47, 85-95.

BRETON Philippe, 2008. Convaincre sans manipuler. Apprendre à argumenter, Paris, La Découverte. 
CHAREST Francine, ALCANTARA Christophe, LAVIGNE Alain et MOUMOUNI Charles (dir.), 2017, E-réputation et influenceurs dans les médias sociaux. Nouveaux enjeux pour les organisations, Québec, Presses de l'université du Québec.

CIALDINI Robert B., 2009, Influence : Science and practice, vol. 4, Boston, Pearson education.

COLLEONI Elanor \& ARVIDSSON Adam, 2014, "Echo Chamber or Public Sphere? Predicting Political Orientation and Measuring Political Homophily in Twitter Using Big Data : Political Homophily on Twitter ", Journal of Communication, 64 : 2, 317-332.

CORDELIER Benoît \& BREDUILLIEARD Pauline, 2012, "Performativité des chartes d'utilisation des réseaux socionumériques en entreprise. Une pragmatique par l'engagement et la contrainte ", Les enjeux de l'information et de la communication, 13 : 1, 127, [https://doi.org/10.3917/enic.012.0009].

CORDELIER Benoît \& DESAULNIERS Audrey-Anne, 2020, "Équilibres et médiations dans la commandite de productions artistiques : Intermédiation formelle ou informelle ", Canadian Journal of Communication, 45, [https://doi.org/10.22230/ cjc.2020v45n1a3447].

CORDELIER Benoît \& TURCIN Karine, 2005, "Utilisations du lien social sur l'Internet comme élément fidélisant à une marque : les exemples de Coca-Cola et d'ESP ", Communication \& organisation, 27, 45-56, [https://doi.org/10.4000/ communicationorganisation.3238].

COTTER Kelley, 2019, "Playing the visibility game : How digital influencers and algorithms negotiate influence on Instagram ", New Media \& Society, 21 : 4, 895-913.

COUTANT Alexandre, 2013, "Quelle place pour l'innovation dans les médias sociaux ?» Communication \& Organisation, 43, 123-134.

DESMOULINS Lucile, ALLOING Camille et MOHLI Vanessa, 2018, "L'influence n'est-elle que donnée(s) ? Médiations et négociations dans les agences de communication «influenceurs» ", Communication \& Organisation, 57, 29-40.

DiSTASO Marcia W. \& BORTREE Denise S. (dir.), 2014, Ethical Practice of Social Media in Public Relations, New York, Routledge.

DUPONT Luc, 2011, 1001 trucs publicitaires (3e édition), Montréal, Transcontinental.

FEICK Lawrence F., PRICE Linda L., HIGIE Robin A., 1986, People who use people: The other side of opinion leadership, ACR North American Advances.

FRANÇOIS Ludovic \& ZERBID Romain (dir.), 2015, Influentia, la référence des stratégies d'influence, Paris, Lavauzelle.

GENDRON Corinne, 2014, "Penser l'acceptabilité sociale : au-delà des intérêts, les valeurs ", Revue internationale de communication sociale et publique [Communiquer], 11.

GRÄVE Jan-Frederik, 2017, « Exploring the perception of influencers vs. traditional celebrities: are social media stars a new type of endorser ? ", Proceedings of the 8th international conference on Social Media \& Society, 1-5. 
HAMPTON Keith N., SHIN Inyoung, WEIXU Lu, 2017, « Social media and political discussion : when online presence silences offline conversation ", Information, Communication \& Society, 20 (7), 1090-1107.

HEATH Robert L., 2006, "Onward Into More Fog: Thoughts on Public Relations' Research Directions ", Journal of Public Relations Research, 18 : 2, 93-114.

HUFFAKER David, 2010, "Dimensions of leadership and social influence in online communities ", Human Communication Research, 36 : 4, 593-617.

JODELET Denise, 2003, Les représentations sociales, Paris, PUF.

JUANALS Brigitte \& MINEL Jean-Luc, 2013, "Construction d'une approche interdisciplinaire et expérimentale pour l'analyse de la communication d'influence ", ESSACHESS-Journal for Communication Studies, $6: 1$ (11), 187-200.

KAPFERER Jean-Noël, 1978, Les chemins de la persuasion : le mode d'influence des media et de la publicité sur les comportements, Paris, Dunod.

KATZ Elihu \& LAZARSFELD Paul F., 1955, The Part Played by People in the Flow of Mass Communication, New York, The Free Press.

KONDRATOV Alexander, 2018, "Stratégies de résistance des professionnels de relations publiques à l'utilisation des Big Data dans les organisations en France et en Belgique : logiques, causes et motifs de non-usage des données numériques massives ", Communication \& Organisation, 57, 121-132.

KOUTROUBAS Theodoros \& LITS Marc, 2011, Communication politique et lobbying, Bruxelles, De Boeck Supérieur.

LALANCETTE Mirelle, YATES Stéphanie \& ROUILLARD Carol-Ann, 2020, " \#Participating \#Contesting: Studying Counterpublics' Discourses on Twitter About the Social Acceptability of Medical Assistance in Dying Legislation in Canada ", Canadian Review of Sociology/Revue canadienne de sociologie, 57 : 4, 604-631, [https:// doi.org/10.1111/cars.12303].

LOCK Irina, SEELE Peter \& HEATH Robert L., 2016, "Where Grass Has No Roots: The Concept of "Shared Strategic Communication" as an Answer to Unethical Astroturf Lobbying ", International Journal of Strategic Communication, $10: 2$.

MAIGRET Éric, 2015, Sociologie de la communication et des médias (3 édition), Paris, Armand Colin.

MASSÉ Guy, MARCON Christian \& MOINET Nicolas, 2006, «Les fondements de l'intelligence économique : réseaux \& jeu d'influence ", Market Management, $6: 3$, 84-103.

MOTULSKY Bernard, 2018, "Les relations de presse ", in S. Yates, Introduction aux relations publiques. Fondements, enjeux et pratiques, Québec, Presses de l'université du Québec, 231-252.

MUCCHIELLI Alex, 2009, L'art d'influencer, Paris, Armand Colin.

OUIMET Gérard, 2008, Psychologie du pouvoir organisationnel. La maîtrise des habiletés politiques, Montréal, Chenelière éducation. 
POELL Thomas, RASHA Abdulla, BERNHARD Rieder, ROBBERT Woltering \& ZACK Liesbeth, 2016, " Protest Leadership in the Age of Social Media ", Information, Communication \& Society, $19:$ 7, 994-1014.

SELTZER Nicholas A., JOHNSON April A., AMIRA Karyn A., 2013, "Revisiting Dynamic Social Impact Theory: Extensions and Applications for Political Science ", International Journal of Politics, Culture \& Society, 26, 349-367.

SINGH Jaywant, CRISAFULLI Benedetta, XUE Melanie T. et al., 2020, "To trust or not to trust: the impact of social media influencers on the reputation of corporate brands in crisis ", Journal of Business Research, 119, 464-480.

TURBIDE Olivier, 2017, "S’excuser publiquement sur les médias socionumériques. Mutation d'une stratégie de gestion de la réputation en communication politique?" ", in M. Burger, Discours des réseaux sociaux : enjeux publics, politiques et médiatiques, Louvain-la-Neuve, De Boeck Supérieur, 141-160.

YATES Stéphanie, 2018, "L'acceptabilité sociale en tant que nouvel impératif des organisations ", in S. Yates (dir.), Introduction aux relations publiques. Fondements, enjeux et pratiques, Québec, Presses de l'université du Québec, 203-227.

YATES Stéphanie, 2019, "The notion of social acceptability: Lay citizens as a new political force ", in M. Lalancette, V. Raynauld \& E. Crandall (éd.), What's \#Trending In Canadian Politics? Understanding Transformations in Power, Media, and the Public Sphere, Vancouver, UBC Press, 256-275.

\section{Biographies des auteurs}

Camille Alloing est professeur en relations publiques à l'Université du Québec à Montréal (UQAM) après avoir été maître de conférences à l'IAE de l'université de Poitiers, ingénieur R\&D et consultant en communication numérique. Il est directeur du Laboratoire sur l'influence et la communication (LabFluens), et chercheur au Laboratoire sur la communication et le numérique (LabCMO). Ses travaux s'intéressent aux mécanismes de production et de circulation de l'information dans les environnements numériques.

Benoit Cordelier est professeur au département de Communication sociale et publique de l'université du Québec à Montréal (UQAM) où il est chercheur au Centre de recherche sur la communication et la santé (ComSanté), à la chaire de relations publiques et communication marketing et directeur de Communiquer Revue de communication sociale et publique. Ses recherches s'articulent principalement autour de deux volets indépendants : un sur la socialisation dans des communautés en ligne et l'autre sur l'analyse de processus organisationnels. 
Stéphanie Yates est professeure au département de Communication sociale et publique de l'université du Québec à Montréal (UQAM) et membre du Groupe de recherche en communication politique de l'université Laval. Politologue de formation (Ph.D université Laval, 2010) et privilégiant une approche interdisciplinaire, elle s'intéresse au lobbyisme, à la participation publique, à l'acceptabilité sociale, à la responsabilité sociale des organisations et à la communication environnementale. 